\title{
The molecular torus in NGC 1052
}

\section{M. Violette Impellizzeri ${ }^{*}$}

Max-Planck-Institute für Radiostronomie, Bonn, Germany

E-mail: violette@mpifr-bonn.mpg.de

\section{Alan L. Roy, Christian Henkel}

Max-Planck-Institute für Radiostronomie, Bonn, Germany

E-mail: aroy@mpifr-bonn.mpg.de, p220hen@mpifr-bonn.mpg.de

The existence of an obscuring torus in the cores of AGN is a key ingredient to the unified scenario, in which a single physical model is used to reproduce radio-quiet type 1 AGN (with broad lines) and type 2 AGN (without broad lines). In the standard picture the torus is an axi-symmetric absorber containing large amounts of molecular gas and dust, and surrounding the accretion disk. Whilst the unified scheme has proven very successful, little is still known about the physical properties of the torus itself. The study of centimetre-wave molecular absorption against strong continuum sources can be used to test these models, but searches for absorption lines of common molecules like $\mathrm{CO}$ and $\mathrm{OH}$ have mostly yielded non-detections. This has lead to the conclusion that the small-scale molecular tori may not exist. Here, we investigate the effects of radiative excitation due to the proximity of a bright radio nucleus, which can suppress the opacity in the lowest transitions causing excitation into higher states. To explore these effects, we conducted a spectralline VLBI observation searching for excited $\mathrm{OH}$ at $13.4 \mathrm{GHz}$ towards the core of NGC 1052 and obtained a detection. These observations confirm the presence of a molecular torus in this source. The absorption profile suggests an obscuration of the inner jet in a compact region confined within $<0.3 \mathrm{pc}$ from the core, which is likely to be associated with a geometrically thick plasma torus and is located $0.1 \mathrm{pc}$ from the eastern jet and $0.7 \mathrm{pc}$ from the western jet.

The 9th European VLBI Network Symposium on The role of VLBI in the Golden Age for Radio Astronomy and EVN Users Meeting

September 23-26, 2008

Bologna, Italy

\footnotetext{
* Speaker.
} 


\section{Radiative excitation and the molecular torus}

\subsection{The molecular torus}

The unified scheme of AGN aims at unifying two or more classes of objects by suggesting that they are a single type of physical object observed from different angles. In this unified scheme, an absorber consisting of parsec-scale molecular gas as well as hot dust surrounds the central engine (e.g. [1]), thus blocking the broad line region (BLR) from direct view. The geometry of the absorber is thought to be either that of a torus or of a warped disk. Indeed, small-scale $(<1 \mathrm{pc})$, clumpy tori have been detected through interferometric infrared studies of the hot dust emission in the cores of AGN (e.g. [2], [3]), and water-vapour masers could map the central few parsecs of active nuclei [ 4 , tracing the rotation of nuclear accretion disks. However, the small thickness of these disks cannot account for the frequently observed spatial obscuration and little evidence has emerged for the larger-scale ( $>1 \mathrm{pc}$ ) molecular components invoked in torus models.

Studies of the molecular absorption and emission can give further clues. The high column densities required for effective obscuration offer us the prospect of finding tori by looking for molecular absorption lines at centimetre wavelengths against a strong nuclear radio synchrotron core. The existence of circumnuclear gas in AGN has been supported by large-scale H I absorption measurements of type 2 radio sources, and observations of CO carried out toward NGC 1068 by e.g. Schinnerer et al. [5] trace rotating molecular clouds with an outer radius of $13 \mathrm{pc}$ from the nucleus. Moreover, from the spectral index distribution, evidence for free-free absorption gives a further indication for obscuration along the line of sight toward the core. Free-free absorption is caused by an ionized region around the central engine, possibly forming an accretion disk or torus.

Despite many efforts to directly detect the expected molecular absorption or emission in a number of surveys, only in very few cases could molecular absorption be confirmed. Single-dish $\mathrm{OH}$ surveys at $18 \mathrm{~cm}$ wavelength of several hundred galaxies revealed $\mathrm{OH}$ absorption towards two Seyferts only and maser emission in five [6], [7], [8]. The lack of absorption in these surveys seems to be in contradiction with the unified scheme, as extragalactic $\mathrm{OH}$ has been shown to display the characteristics of the circumnuclear environment of active galaxies. Tracing the low density component of the molecular environment in the inner few hundred parsecs $\mathrm{OH}$ should therefore be better tracer for the extended molecular torus.

There are a few possible causes for the absence of detectable molecular absorption: (1) the molecular abundances are low due to harsh conditions, (2) the gas temperature and hence state of excitation due to collisional excitation are high enough that the lowest transitions probe an undetectably small fraction of the molecules and (3) the non-thermal continuum radiation of the central source enforces a high excitation temperature, much higher than the kinetic temperature, that suppresses the opacity in the lowest transitions [9]. Before concluding that tori are not molecular, the last of these points, the radiative coupling to the non-thermal continuum, deserves further attention and influences the selection of the most favourable transitions to observe.

\subsection{Radiative excitation in the torus}

Assuming that the ionization parameter within the torus is low enough for the gas to be molecular and if the torus is sufficiently compact, the molecules can radiatively couple to the bright radio continuum source increasing the rotation temperature and lowering the fractional population 
of the low $J$-levels, increasing the excitation temperature of the lower rotational levels, and thus suppressing the absorption in the lower transitions [- $]$ ].

Calculations done for radiative coupling of $\mathrm{OH}$ at $1.67 \mathrm{GHz}$ were carried out by Black [10], showing that $\mathrm{OH}$ suffers similar excitation effects that may make its detection also difficult towards the cores of AGN with strong radio continuum emission. Black [10] calculated that the opacity in the $1.67 \mathrm{GHz}$ transitions of $\mathrm{OH}$ located $10 \mathrm{pc}$ from the AGN will be suppressed by a factor of $10^{3}$ due to the proximity of the bright nuclear radio continuum source. In contrast, the opacity of the $6 \mathrm{GHz}$ transitions will be suppressed by a factor of $10^{2}$, and the opacity of the $13.4 \mathrm{GHz}$ transition will increase slightly by a factor of two. Thus, if the molecular gas is concentrated in a $1 \mathrm{pc}$ to $10 \mathrm{pc}$ scale torus, then radiative excitation effects should be strong at $1.67 \mathrm{GHz}$ and the gas would then be best found by looking at higher-order transitions.

\section{Observations}

To explore these effects we modified the search strategy for molecular absorption, searching for excited $\mathrm{OH}$ at $13.4 \mathrm{GHz}$ in NGC 1052 with the VLBA. NGC 1052 is a prime candidate to search for excited $\mathrm{OH}$, as there is observational evidence for obscuration towards the core.

NGC 1052 is a galaxy with a $13.4 \mathrm{GHz}$ continuum flux density of $1 \mathrm{Jy}$ to $2 \mathrm{Jy}$ arising from a central radio source of size $\sim 10$ mas (see Fig. 1). Free-free absorption was found in the inner few parsecs around the nucleus [11], [12], apparently due to a geometrically-thick, patchy structure oriented roughly orthogonal to the jets. Since the western jet is covered more deeply and extensively, it is thought to be receding. Opacities have been measured implying a dense $\left(n_{\mathrm{e}}=10^{5} \mathrm{~cm}^{-3}\right)$ plasma associated with the central $0.5 \mathrm{pc}[13]$.

VLBA observations with the aim of looking for the highly excited ${ }^{2} \Pi_{\frac{3}{2}}, J=\frac{7}{2}$ states of $\mathrm{OH}$ ( $290 \mathrm{~K}$ above the ground-level) at $13441 \mathrm{MHz}(\mathrm{F}=4-4)$ and $13435 \mathrm{MHz}(\mathrm{F}=3-3)$ were made on December 6 and 8, 2005 for $7 \mathrm{~h}$ each day (including observations of the calibrators). Observations were carried out with four IFs to observe two polarizations with $16 \mathrm{MHz}$ bandwidth, corresponding to $357 \mathrm{~km} \mathrm{~s}^{-1}$, per IF. The IFs were centred at the source frame rest frequencies of $13.434 \mathrm{GHz}$ and $13.441 \mathrm{GHz}$, providing a total bandwindth of $19 \mathrm{MHz}$ and $13 \mathrm{MHz}$ of overlap. With 256 channels per IF, a spectral line velocity resolution of $1.4 \mathrm{~km} \mathrm{~s}^{-1}$ per channel was obtained. The total velocity range was $700 \mathrm{~km} \mathrm{~s}^{-1}$ when two IFs were placed adjacent to each other to form a single spectrum, which allowed one third of the channels to measure the line-free continuum level. The data were calibrated using standard phase and amplitude calibration, using 0423-01 and 3C 84 for bandpass calibration.

\section{Results and discussion}

The continuum image of NGC 1052 from the VLBA observations is shown in Fig. 1. At $13.4 \mathrm{GHz}$ the source structure is extended, with jets extending east-west up to $\sim 1 \mathrm{pc}$ from the core, although some low-level emission was seen further out extending up to $\sim 2$ pc from the core. The maximum peak flux density of the source is $436 \mathrm{mJy}_{\text {beam }}^{-1}$, the noise in the map is $0.7 \mathrm{mJy}_{\text {beam }}{ }^{-1}$. Absorption of the rotationally excited $\mathrm{OH}$ transition at $13.434 \mathrm{GHz}$ was clearly detected towards the centre of NGC 1052. Absorption was detected predominantly towards the 


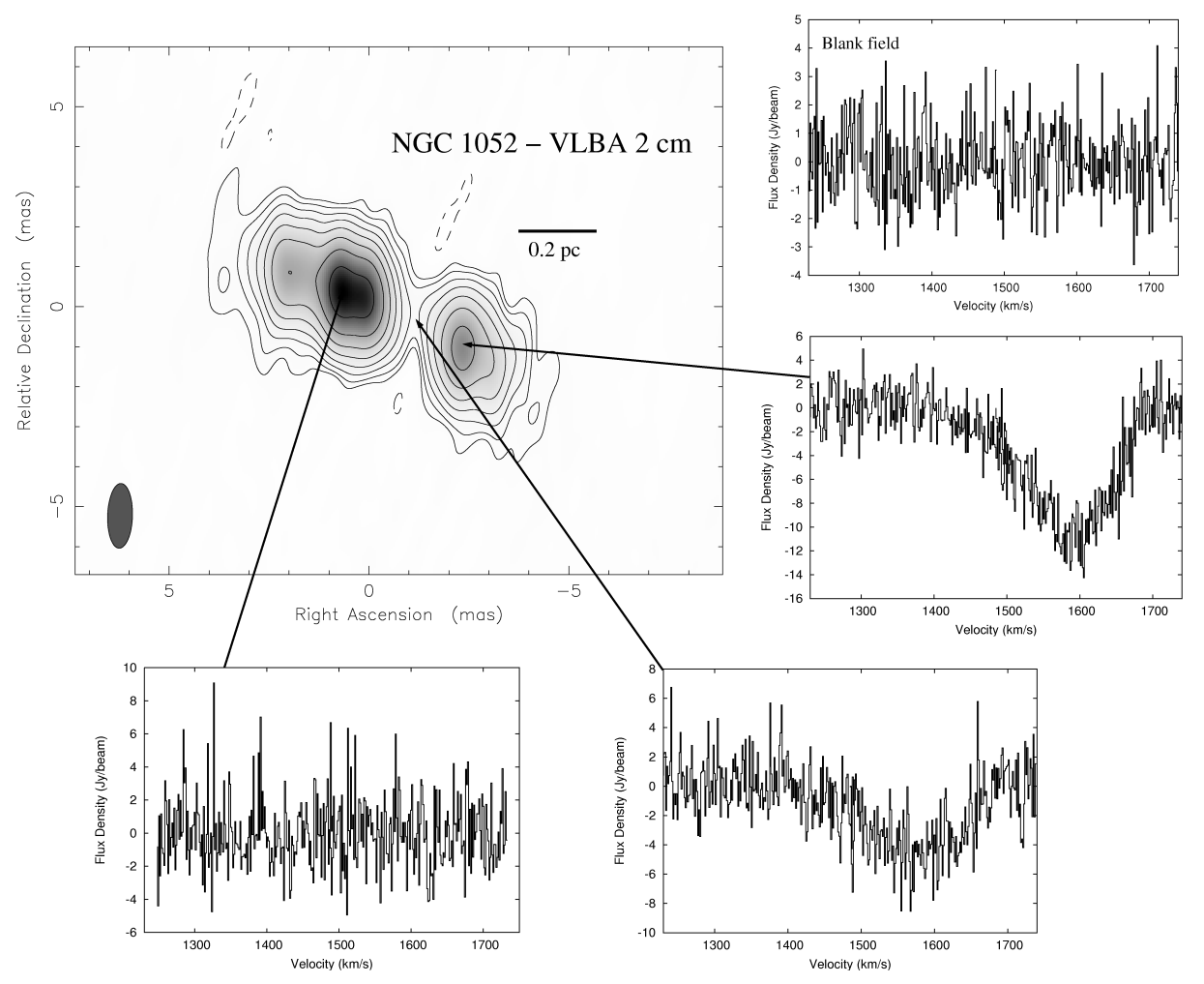

Figure 1: VLBA image of NGC 1052 at $13.4 \mathrm{GHz}$. The synthesized beam is $(1.62 \times 0.62) \mathrm{mas}^{2}$. The image is contoured at $-3,3,6,12,24,48,96$ times the map noise of $0.7 \mathrm{mJy}_{\text {beam }}{ }^{-1}$. The maximum in the map is $0.436 \mathrm{Jy} \mathrm{beam}^{-1}$. The panels show a montage with selected $\mathrm{OH}$ absorption spectra. The top right panel shows the absorption spectrum integrated over a blank field off the source.

western jet (the counter-jet), whereas none was found towards the eastern jet. The apparent optical depth of the spectrum taken towards the counter-jet derived from the ratios of intensities of peak absorption and adjacent continuum is $\tau \sim 0.26$ with a line FWHM corresponding to $\sim 200 \mathrm{~km} \mathrm{~s}^{-1}$. Towards the "gap" between the jet and the counter-jet, the apparent optical depth of the line was $\tau \sim 0.23$. Although the absorption line has a line flux density of $-4.8 \mathrm{mJy}$ at the gap which is less than the line flux density at the counter jet, $-11.2 \mathrm{mJy}$, the optical depths are comparable in the two regions, due to weaker continuum in the gap.

The absorption profile observed with the VLBA suggests an obscuration of the inner jet region in an extremely compact region confined within a distance $<0.3 \mathrm{pc}$ from the core. The proximity to the core is further supported by the large line width. The line is relatively broad compared to the $\mathrm{OH}$ absorption spectrum associated with the nuclear region observed at $1.67 \mathrm{GHz}$ [14], [12]. Also, $\mathrm{H} \mathrm{I}$ absorption, with different velocity components (a high velocity system and sharper features at low velocity), has been associated with the central few pc region. The $1.67 \mathrm{GHz} \mathrm{OH}$ and $\mathrm{H}$ I gas are thought to be co-located due to the similarity of their absorption profiles. $\mathrm{CO}, \mathrm{HCN}$, and $\mathrm{HCO}^{+}$ have been detected in absorption in this source [15], with line profiles that are broader than the $\mathrm{H} \mathrm{I}$ and $1.67 \mathrm{GHz} \mathrm{OH}$ profiles, and are more similar to our $13.4 \mathrm{GHz}$ spectrum. The broader widths of these lines are due to lower free-free opacity at mm-wavelengths which exposes higher-velocity material nearer to the nucleus. The different velocity peaks at the different transitions trace slightly 
different components of the nuclear region. However, they share a common overall velocity range. NGC 1052 is also an $\mathrm{H}_{2} \mathrm{O}$ megamaser galaxy. The maser originating at $0.1 \mathrm{pc}$ to $0.2 \mathrm{pc}$ to the west of the core in the counterjet is thought to be excited by an interaction of the jet with the surrounding medium [16]. The positions of the $\mathrm{H}_{2} \mathrm{O}$ masers are coincident with the position at which the $\mathrm{OH}$ absorption presented here is observed, and it seems likely that these molecules, and the gas observed at mm-wavelength coexist.

Free-free absorption in NGC 1052 leads to an asymmetric central gap that opens up towards lower frequencies. The western presumably receding jet is partially obscured by free-free absorption, at least in the inner parsec. The free-free absorption towards the western jet and the gap between eastern and the western jet have been explained with the presence of a geometrically thick plasma torus and a geometry of the jet-torus system in which $0.1 \mathrm{pc}$ of the eastern jet and $0.7 \mathrm{pc}$ of the western jet are obscured [11]. The $\mathrm{OH}$ absorption observed here confirms this expectation and appears to be associated with the inferred torus in this source. The H I atomic gas absorption, on the other hand, is distributed in front of the approaching, as well as the receding, jet and has sub-pc scale structure. While the $\mathrm{OH}$ gas at $1.67 \mathrm{GHz}$ and the $\mathrm{H}$ I gas are likely to be co-located, they do not seem to coincide with the $\mathrm{H}_{2} \mathrm{O}$ masers, even though these are at the same velocity [16]. Although the apparent optical depth of the $1.67 \mathrm{GHz}$ line was measured to be $\tau=0.25$, and is comparable to the optical depth of the $13.4 \mathrm{GHz}$ line, these $\mathrm{OH}$ lines probably arise in different regions, that is, we are here tracing gas which is more compact and closer to the nuclear engine. It is remarkable how in the inner parsec around the AGN it appears possible for some molecular and atomic clouds to preserve a relatively quiescent existence.

\section{Conclusions}

We report here the detection of excited $\mathrm{OH}$ at $13.4 \mathrm{GHz}$ towards the core of NGC 1052. Absorption was observed towards the western jet of the source, whereas none was found towards the eastern jet. The optical depth of the absorption line was $\tau \sim 0.26$ with a line FWHM of $\sim$ $200 \mathrm{~km} \mathrm{~s}^{-1}$. The absorption profile suggests obscuration of the inner jet in a compact region confined within $<0.3 \mathrm{pc}$ from the core. The vicinity to the core is further supported by the large width of the line. The gas is likely to be associated with a geometrically thick plasma torus, which has been inferred from free-free absorption and is located $0.1 \mathrm{pc}$ from the eastern jet and $0.7 \mathrm{pc}$ from the western jet. Also, $\mathrm{OH}$ seems to be co-located with the $\mathrm{H}_{2} \mathrm{O}$ gas and perhaps with the more compact clouds of $\mathrm{CO}, \mathrm{HCN}$, and $\mathrm{HCO}^{+}$, but not with the $\mathrm{HI}$ and $\mathrm{OH}$ gas observed at $1.67 \mathrm{GHz}$, which is more evenly distributed around the nuclear region.

These observations confirm that radiative excitation effects and higher-order transitions should be taken into account when studying the molecular properties in the innermost regions of AGN. The detection of excited $\mathrm{OH}$ at $13.4 \mathrm{GHz}$ provides independent evidence for a molecular torus at the centre NGC 1052 on parsec scale.

\section{References}

[1] J.H. Krolik; M.C. Begelman, Molecular tori in Seyfert galaxies - Feeding the monster and hiding it (1988) Ap J 329, 70 
[2] W. Jaffe; K. Meisenheimer; H. Röttgering et al., The central dusty torus in the active nucleus of NGC 1068 (2004 ) nature 02531

[3] K.R.W. Tristram; K. Meisenheimer; W. Jaffe et al.,Resolving the complex structure of the dust torus in the active nucleus of the Circinus galaxy (2007) A\&A) $\mathbf{4 7 4} 837$

[4] J.R. Herrnstein; J.M. Moran; L.J. Greenhill et al., A geometric distance to the galaxy NGC4258 from orbital motions in a nuclear gas disk (1999) nature 400539

[5] E. Schinnerer; A. Eckart; L.J Tacconi et al., Bars and Warps Traced by the Molecular Gas in the Seyfert 2 Galaxy NGC 1068 (2000) ApJ 533850

[6] J.T. Schmelz; W.A. Baan; A.D. Haschick et al., An Arecibo survey for extragalactic hydroxyl absorption. I - Presen tation of results, (1986) AJ 92, 1291

[7] W.A. Baan, Willem; A. Haschick et al. Hydroxyl in galaxies. I - Surveys with the NRAO 300 FT telescope (1992 AJ, 103, 728

[8] L. Staveley-Smith; R.P. Norris; J.M. Chapman et al. A southern OH megamaser survey (1992) MNRAS 258, 725

[9] P. Maloney; M. C. Begelman; M. J. Rees, Radiative excitation of molecules near powerful compact radio sources (199 4) ApJ, 432, 606

[10] J. Black, The Molecular Astrophysics of Stars and Galaxies (1998), 469

[11] S. Kameno; S. Sawada-Satoh; M. Inoue et al. The Dense Plasma Torus around the Nucleus of an Active Galaxy NGC1052 (2001) PASJ 53169

[12] R.C. Vermeulen; E. Ros; K.I. Kellermann et al. The shroud around the twin radio jets in NGC 1052 (2003) A\&A 401113

[13] M. Guainazzi; T. Oosterbroek; L.A. Antonelli et al. On the hypothesis of an advection-dominated flow in the core of NGC 1052: new constraints from a BeppoSAX observation (2000) A\&A 36480

[14] A. Omar; K.R. Anantharamaiah; M. Rupen et al. VLA detection of OH absorption from the elliptical galaxy NGC 1052 (2002) A\&A 38129

[15] H. Liszt; R. Lucas Mm-wave HCO+, HCN and CO absorption toward NGC 1052 (2004) A\&A 428 445

[16] M.J. Claussen; P.J. Diamond; J.A. Braatz The Water Masers in the Elliptical Galaxy NGC 1052 (1998) ApJ 500129 